\title{
XXXII.
}

\section{Analyse der Samen des weissen Mohns.}

\author{
Von \\ Prof. Sace.
}

(Ann. de chim. et de phys. $X X 11,473$.

Die Analyse der Samen des weissen Mlohns wurde in drei Theile getheilt, deren Resultate sich gegenseitig controliren. Der erste Theil umfasst die näberen Bestandtheile der Samen der zweite die entfernteren Bestandtheile und der dritte die Bestimmung der Asche und die Analyse derselben.

\section{Analyse der näheren Bestandtheile.}

Die angewendeten Samenkörner waren sehr rein und weiss; sie waren im Jahre 1838 in einem Kalkmergelboden gewachsen. Die Ernte war eine sehr schöne und die Pflanzen waren kräftig.

Da diese Samenkörner wegen ihrer grossen Elasticität, die sich dem Pulvern in einem Mörser widersetzte, nicht direkt analysirt werden konnten, wurde das 0el durch Pressen daraus gewomnen. 24 Kilogrm. Samenkörner gaben 10,50 Kilogrm. reines 0el; 15,2049 Kilogrm. rohen 0elkuchen, entsprechend 12,773 Kilogrm. trocknen, und 0,727 liilogrm. in den Körnern enthaltenes Wasser, das durch den Verlust ermittelt wurde. Das Auspressen geschah zuerst in der Kälte, und dann mit einem Zusatz von bis auf $30^{\circ} \mathrm{C}$. erwärmtem Wasser. Dieser zuletzt gewonnene Antheil wurde, als möglicherweise verändert, nach dem Wägen bei Seite gesetzt.

Um die Menge des in dem Oelkuchen enthaltenen Wassers zil erfahren, wurden drei Portionen des Kuchens genommen, und über dieselben trocknes Kohlensäuregas bei $100^{\circ}$ geleitet, his keine Gewichtsabnahme mehr stattfand. Diese Vorsichtsmassregel wurde bei allen Wasserbestimmungen, von denen in der Folge die Rede sein wird, angewendet; sie war nothwendig wegen der grossen Menge Sauerstoff, welche das Mohnōl in kurzer Zeit absorbirt.

I. 7,9308 Grm. roher 0elkuchen wogen nach dem Trocknen 6,6617 Grm.

II. 7,5155 Grm. derselben Substanz gaben 6,3128 Grm. trocknen Delkuchen. 
III. 8,8272 Grm. gaben 7,4368 Grm. trockne Sulstanz. Diese Resultate geben in $\mathbf{1 0 0}$ Theilen:

\begin{tabular}{|c|c|c|c|c|}
\hline \multirow{3}{*}{$\begin{array}{l}\text { Feste Substanz } \\
\text { Wasser }\end{array}$} & 830.855 & II. & III. & Im Mittel. \\
\hline & $\begin{array}{l}83,9085 \\
16,0015\end{array}$ & $\begin{array}{l}83,9970 \\
16,0030\end{array}$ & $\begin{array}{l}84,0229 \\
15,9779\end{array}$ & $\begin{array}{r}84.0059 \\
15.9941\end{array}$ \\
\hline & $\overline{100,000}$ & $\overline{100}, \overline{0000}$ & $100, \overline{0000}$ & 100.0000 \\
\hline
\end{tabular}

Wälırend der ersten Stunden des Austrocknens zeigte der aus dem Rohr entweichende Kohlensäurestrom einen faden und unangenehmen Geruch, der an den des Mohnöls erinnert; er scheint von der Gegenwart eines flüchtigen, fetten Körpers herzurühren, der sich in dem 0 el in kleiner Menge findet.

Der Oelkuchen enthielt noch Oel; er wurde in dem Verdrängungsapparate von Pelouze mit Aether behandelt, bis die ablaufende Flüssigkeit nach dem Abdampfen keinen merklichen Rückstand mehr hinterliess.

126 Grm. Oelkuchèn, 105,8474 Grm. getrockneten entsprechend, hinterliessen einen in Aether unlöslichen Rücksland, der $115 \mathrm{Grm}$. wog. Es wurden von demselben zwei Portionen getrocknet.

I. 3,6915 Grm. gaben 2,8163 Grm. trocknen Rückstand;

II. 4,3240 Grm. gaben 3,2940 Grm. trocknen Rückstand. In 100 Theilen:

\begin{tabular}{lccc} 
& I. & II. & In Mittel. \\
Nicht flüchtige Substanzen & 76,2914 & $\mathbf{7 6 , 1 7 9 4}$ & $76, \mathbf{2 3 5 4}$ \\
Flüchtige Substanzen & $\mathbf{2 3 , 7 0 8 6}$ & $\mathbf{2 3 , 8 2 0 b}$ & $\mathbf{2 3 , 7 6 4 0}$ \\
\cline { 2 - 4 } & $\mathbf{1 0 0 , 0 0 0 0}$ & $\frac{100,0000}{100,0000 .}$ & $\frac{10000}{}$
\end{tabular}

Der Gesammtrückstand hàte demnach $80,6931 \mathrm{Grm}$. wiegen müssen ; er war gelblich weiss, geruch- und geschmacklos.

Die atherische Lösung war vollkommen hell und grünlich-

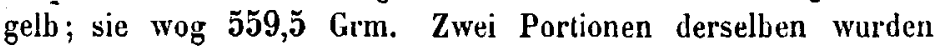
über Chlorcalcium im leeren Raume getrocknet; der Rückstand war von Oelconsistenz, und durch einige kleine Nadeln einer stearoptenähnlichen Substanz getrübt. Dieser Rückstand besass im hohen Grade den zu gleicher Zeit faden und scharfen Geschmack des Oeles, dessen riechendes Princip in der Hülle und nicht in dem Mark der Körner seinen Sitz hat; es scheint demnach ein ätherisches $0 \mathrm{el}$ zu sein.

1. 4,4233 Grm. ätherische Lõsung hinterliessen 0,1449 Rūckstand ;

II. 5,4215 Grm. ătherische Lősung hinterliessen 0,1774 Rūckstand. 
Diese Resultate geben in 100 Theilen:

\begin{tabular}{|c|c|c|c|}
\hline \multirow[t]{2}{*}{$\begin{array}{l}\text { Nicht flüchtige Substanzen } \\
\text { Flüchtige Substanzen }\end{array}$} & $\begin{array}{r}1 . \\
3,2758 \\
96.7242 \\
\end{array}$ & $\begin{array}{c}\text { II. } \\
3,27 \pm 1 \\
96.7279\end{array}$ & $\begin{array}{c}\text { Im Mittel. } \\
3,2739 \\
96,7261\end{array}$ \\
\hline & $\overline{10.0000}$ & $100,0(001)$ & $\overline{100,0000}$ \\
\hline
\end{tabular}

Die Gesammtmenge der ãtherischen Lōsung enthält demnach 18,3174 Grm. Oel und andere in Aether lösliche Substanzen, wie Stearopten, ātherisches Oel und färbende Substanz.

Zieht man von dem Gewicht des trocknen Oelkuchens das des mit Aether behandelten und darauf getroclineten ab, so findet man eine Differenz von 25,1543 Grm., welche $Z$ ahl dem Gewichte der durch Aether entzogenen Substanzen gleich ist. Da die directe Bestimmung aher nur 18,3174 Grm. gab, so existirt zwischen den beiden Bestimmungen ein Unterschierl von 6,8368, der nur von der Verflüchtigung eines älherischen 0eles während des Austrocknens herrühren kann. Die Resultate dieser Analyse müssen daher auf folgende Weise geschrieben werden.

$$
\begin{array}{lr}
\text { Fettes Oel } & 18,317 t \\
\text { Flïchtige Substanzen } & 6,836 y \\
\hline 25,1543 .
\end{array}
$$

Der getrocknete Oelkuchen enthält demnach in 100 Theilen :

$\begin{array}{lr}\text { In Aether unlösliche Substanz } & 76,2353 \\ \text { Fettes Oel } & 17,3055 \\ \text { Flïchtige Substanzen } & 6,7532 \\ & 100,0000 .\end{array}$

Ich prüfte in der von der Behandlung des Oelkuchens herrübrenden ätherisclıen Lōsung auf die im Opiun vorkommenden Pflanzenhasen, aber vergeblich. Die Samenkörner des Mohns sind demnach nicht schädlicher als das aus denselben gewonnene $0 \mathrm{el}$; in Deutschland und in der Schweiz wendet man bekanntlich auch die Mounsamen in der Kuchenbäckerei an. Die Analyse des Oelkuchens zeigt, dass dieses Nahrungsmittel in jeder Beziehung empfohlen zu werden verdient.

Nachdem die in Aether loslichen Bestandtheile der Körner bestimmt worden sind, wurden die den Rückstand bildenden untersucht; derselbe besteht aus einer Pektinverbindung, welche die Eigenschaften des Bassorins zeigt, aus Holzfaser, Casein und einer beträchtlichen Menge von Albumin. Um die Menge der Pektinverbindung $z u$ bestimmen, wurde der rohe Kuchen in 
Gläser mit engem Ilals gebracht, mit der zelınfachen Menge Wasser und einigen Tropfen Schwefelsäure übergossen; die Gläser wurden bei gelinder Wärme im Sandbade acht und vierzig Stunden lang erwärmt, nach Verlauf derselben bis zum Sieden des Inhaltes erhitzt, letzteres auf ein gewogenes Filter gebracht und sorgfältig gewaschen. Der Rückstand wurde getrocknet, gewogen, und 24 Stunden lang bei $35^{\circ}$ mit einer verdünnten Natronlōsung behandelt. welche die Proteïnsubstanzen und das Oel auflöste; der Rückstand zeigte nach dem Waschen alle $\mathrm{Ei}$ genschaften der Holzfas'r.

1. 2,7611 Grm. trockner Kuchen linterliessen nach dem Behandeln mit Schwefelsäure einen Rückstand von 1,5907.

II. 2,0190 Grm. Substanz gaben 1,1633 Grm. Rückstond. In 100 Theilen:

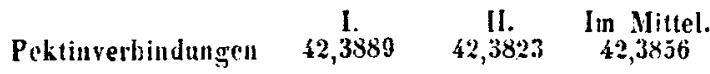

Weun man den Oelkuchen mil verdünnter Schwefelsäure behandelt, so findet eine reichliche Entwickelung ron riechenden Substanzen statt; es ist daher sehr wahrscheinlich, dass die Bestimmung der Pektinverbindungen durch Differenz um das ganze Gewicht der flüchtigen Substanzen zu lioch ist. Dic Gesanmtmenge der fetten und riechenden Körper wurle rom Gewichte des Rückstandes, vor der Behandlıng des Kuchens mit Schwefelsäure, nach dem Waschen mit Natron abgezogen; es kann also wohl sein. dass das Gewicht der Proteinsubstanzen etwas zu niedrig ausgefallen ist.

I. 1,0770 Grm. des mit Schwelelsäure behandelten Kuchens, hinterliessen nacb der Digestion nit Natron, einen Rückstand von $0,1978 \mathrm{Grm}$. Gi'm.

Il. 0,6552 Grm. derselben Substanz hinterliessen 0,1255

Die Differenz 0,8792 Grm. des ersten Versuchs entspricht $0,4349 \mathrm{Grm}$. der Proteĩnsubstanzen und $0,4443 \mathrm{Grm}$. der fetten Körper.

Die Differenz 0,5297 des zweiten Versuches entspricht 0,2594 Grm. der Proteinsubstanzen und $0,2703 \mathrm{Grm}$. der fetlen horper. Der mil Schwefelsāure behandelte Kuchen enthält demnach in 100 Theilen an fetten Korpern: 
300 Sace: Analysedes Samens des weissen Molens.

und an Proteïnverbindungen

$$
\stackrel{1 .}{41,2535} \quad \text { II. } \quad \text { In Mittel. }
$$

$$
\begin{array}{lcc}
\text { I. } & \text { II. } & \text { Im Mittel. } \\
40,3807 & 39,5910 & 39,9858
\end{array}
$$

Der Rückstand von beiden Operationen war reine, vollkommen weisse Holzfaser, von eigenthümlichem Atlasglanz.

I. 1,0770 Grm. gaben 0,1978 Grm. Holzfaser.

II. 0,6552 Grm. gaben 0,125j Grm. IIolzfaser.

100 Theile des mit Schwefelsäure und Natron behandelten Kuchens enthalten:

$$
\text { Holzfaser } \quad 18,3658 \quad 19,1545 \quad \text { Im Mittel. }
$$

Durch die Analyse der näheren Bestandtheile stellte sich

\begin{tabular}{|c|c|}
\hline \multicolumn{2}{|l|}{ Fettes 0el, direkt bestimmt } \\
\hline Fettes Oel, durch tether ansgezogen & 4,4479 \\
\hline Flüchtiure Substanzen & $3,5,30$ \\
\hline Pektinverbill dungen & 23.2636 \\
\hline Proteinverbindungen & 12,6148 \\
\hline Holzfaser & 5,9321 \\
\hline & {$[10$} \\
\hline
\end{tabular}
die Zusammensetzung der Körner des weissen Mohns auf folgende Weise heraus. Das hygroskopische Wasser, das hei der Analyse 3,0292 p. C. betrug war abgezogen worden:

Anulyse der entfrnteren Besiandtheile.

Lim das hygroskopische Wasser der rohen Körner zu bestimmen, wurden dieselben bei $100^{\circ}$ in einen Strom trocknem Kohlensäuregas getrocknet und das Wasser durch Differenz bestimmt.

I. 7,9416 Grm. rohe Körner gaben 7,3963 Grm. trockne hörner.

Il. 5,3980 Grm. rohe Körner gaben 5,0273 Grm. trockne Körner.

ln 100 Theilen:

$$
\text { Wasser } \quad \underset{6,8563}{\text { I. }} \quad \underset{6,8673}{\text { II }} \underset{6,8618}{\text { In wiltel. }}
$$

Bei der Analyse im Grossen waren nur 3,0292 p. C. Wasser gefunden werden, d. h. ungefähr die Hälfte weniger, als die direkte Bestimmung gegeben halte. Da die Wägungen mit grosser Sorgfalt vorgenommen worlen waren, so war es unmöglich ilınen diese Differenz zuzuschreiben; sie mochte vielmehr ihren 
Grund darin haben, dass die Mohnkürner ihr hygroskopisches Wasser aufnehmen und wieder algegeben. Es ist dies in so hohem Grale der Fall, dass 4,1709 Grm. trockne Korner, die an einem feuchten Tag, im Laboratorium unter einer Glocke aufbewahrt worden waren, nach sechs Stunden $4,3246 \mathrm{Grm}$. wogen. Sie hatten in dieser kurzen Zeit 0,1537 Grm. oder 3,685 p. C. Wasser aufgenommen.

Die folgenden Analysen wurden mit bei $100^{\circ}$ in einem Strom von trocknem Kohlensüuregas getrockneten Produkten vermittelst chromsaurem Bleioxyd vorgenommen.

I. 0,2414 Grm. der Kürner gaben 0,5̃50 Grm. Kohlensäure und 0,1989 Grm. Wasser.

II. 0,2779 Grm. der Körner gaben 0,6349 Grm. Kohlensäure und 0,2318 Grm. Wasser.

Diese Zahlen geben in 100 Theilen:

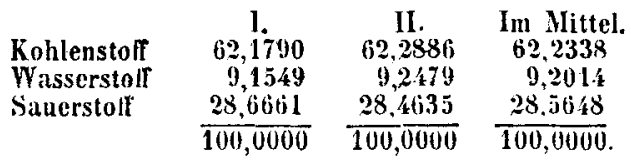

Die Bestimmung des Stickstoffs in den Körnern wurde nach der Nethode von Will und Varrentrapp vorgenommen.

I. 0,4047 Grm. gaben 0,1020 Grm. Platin.

II. 0,3998 Grm. gaben 0,1020 Grm. Platin.

In 100 Theilen:

\begin{tabular}{|c|c|c|}
\hline & I. & II. \\
\hline
\end{tabular}

\section{Verbrennung des rohen Kuchens.}

I. 0,6145 Grm. Kuchens gaben $1,0728 \mathrm{Grm}$. Kohlensäure und $0,3672 \mathrm{Grm}$. Wasser.

II. 0,4509 Grm. Substanz gaben 0,7905 Grm. Kohlensäure und $0,3120 \mathrm{Grm}$. Wasser.

III. 0,3931 Grm. Substanz gaben 0,6894 Grm. Kohlensãure und $0,2378 \mathrm{Grm}$. Wasser.

In 100 Theilen:

\begin{tabular}{|c|c|c|c|c|}
\hline $\begin{array}{l}\text { Kohlenstoff } \\
\text { Wasserst off } \\
\text { Sauerst off }\end{array}$ & $\begin{array}{r}\text { I. } \\
47,3997 \\
6,6395 \\
45,7608 \\
100,0000\end{array}$ & $\begin{array}{r}\text { II. } \\
47,8155 \\
6,9195 \\
45,2650 \\
100,0000\end{array}$ & $\begin{array}{r}\text { III. } \\
47,8250 \\
6,7158 \\
45,4592 \\
100,0000\end{array}$ & $\begin{array}{c}\text { Im Mittel: } \\
47,7468 \\
6,7582 \\
45,4950 \\
100,0000\end{array}$ \\
\hline
\end{tabular}


302 Sace: Analyse des Samens des weissen Mohns.

1. 0,7751 Grin. Kuchen gaben 0,3224 Grm. Platin.

II. 0.6826 Grm. Sulıstanz gaben 0,2902 Grm. Platin.

In 100 Theilen:

$$
\text { Stickstoff } \quad \underset{5,9089}{\text { I. }} \quad \underset{6,0357}{\text { II. }} \underset{5,9723}{\text { Im Mittel. }}
$$

Verbrennung des durch Aether gereinigten Kuchens.

I. $0,2399 \mathrm{Grm}$. Kuchen gaben 0,3718 Grm. Kohlensäure und $0,1301 \mathrm{Grm}$. Wasser.

II. $0,2072 \mathrm{Grm}$. Substanz gahen 0,3212 Grm. Kohlensäure und 0,1137 Grm. Wasser.

In 100 Theilen:

$$
\begin{array}{lrrr} 
& \multicolumn{1}{c}{\text { I. }} & \multicolumn{1}{c}{\text { II. }} & \multicolumn{1}{c}{\text { Im Mittel. }} \\
\text { Kohlenstoff } & 42,2676 & \mathbf{4 2 , 2 7 8 0} & \mathbf{4 2 , 2 7 2 8} \\
\text { Wasserstoff } & 6,0025 & 6,0811 & 6,0418 \\
\text { Sauerstoff } & 51,7299 & 51,6409 & 51,6854 \\
& \frac{50,0000}{100,0000} & \frac{100,0000}{100}
\end{array}
$$

I. 0,3682 Grm. gaben 0,2015 Grm. Platin.

II. 0.2925 Grm. gaben 0.1553 Grm. Platin.

In 100 Theilen:

$$
\text { Slickstor } \quad \underset{7,7675}{7,5213} \quad \underset{7,6444}{\text { Il. }}
$$

\section{Verbrennung des Mohnöls.}

So wie das Oel die Presse verliess, wurde es ror dem Z1tuitt der Luft geschützt, durch Fliesspapier filtrirt und in hermetisch verschlossenen Gefässen aufbewahrt. Es war gelb, vollkommen hell, fast farblos, ein wenig klebrig und von einem schwachen Geruch, der an den des Nussőles erinnerte. Es verseifte sich sowohl vermittelst Alkalien, als auch vermittelst Schwefelsăure mit der grössten Leichtigkeit und gab mit Natron eine harte Seile, deren Weisse die der bessten Olivenölseifen bei weitem übertraf. Da sich diese Seife, selhst wenn sie sehr viel Alkali enthält, an der Luft nicht verändert, so kann man daraus schliessen, dass die trocknende Oelsăure des Mohnöles eine andere als die des Leinöles sei.

Nachdem ich mich überzeugt hatte, dass das angewendete Oel wicht die geringste Spur Wasser enthielt, setzte ich 10,9916 Grm. der Einwirkung eines bis auf $100^{\circ}$ erhitzten Stromes von trocknem Kohlensãuregas aus. Nach Verlauf von zwei Stunden 
liatte das Oel kein Wasser verloren, und sein Gewicht um $0,0193 \mathrm{Grm}$. zugenommen, was von einer Absorption des Sauerstoffs herrührte, die während des Eingiessens des 0eles in den Trockenapparat und des Ausgiessens nach beendigter Operation stattgefunden hatte. Dieses Faktum beweisst, dass die Maler recht haben, wenn sie sagen, dass das Mohnöl bei weitem leichter trockne als das Leinöl. Das Mohnǒl ist vollkommen neutral und enthält kein Ammoniak.

I. 0,3547 Grm. Oel gaben 0,9982 Grm. Kohlensăure und 0,3673 Grm. Wasser.

II. 0,2836 Grm. gaben 0,7971 Grm. Kohlensãure und $0,2978 \mathrm{Grm}$. Wasser.

III. 0,2466 Grm. gaben 0,6920 Grm. Kohlensăure und 0,2615 Grm. Wasser.

In 100 Theilen:

\begin{tabular}{lcccc} 
& I. & II. & III. & Im Miltel. \\
Kohlenstof & 76,7409 & 76,6220 & 76,5207 & 76,6279 \\
Wasserstoff & 11,5027 & 11,6362 & 11,7599 & 11,6329 \\
Sauerstoff & 11,7564 & $\frac{11,7418}{11,7194}$ & 11,7392 \\
\cline { 2 - 5 } & $\frac{11,7000}{100,0000}$ & $\frac{100,16000}{100,0000 .}$
\end{tabular}

Um die vorstehenden Analysen so genau als möglich zu machen, wurde versucht den Sauerstoff der organischen Substanz vermittelst chromsaurem Baryt zu bestimmen, das vor und nach der Verbrennung analysirt die zur Umwandelung der organisirten Substanz in Gas nothwendige Menge Samerstoff verloren haben musste. Die Verbrennung geht eben so leicht mit dem chromsauren Baryt als mit dem chromsauren Bleioxyd vor sich. Man erbält die Gesammtmenge des Wassers, aber nie melır ats drei Viertel der Kohlensäure. Der chromsaure Baryt muss deshalb in den Laboratorien zu den Verbrennungen verworfen werden, in den Vorlesungen ist aber diese Substanz sehr nützlich, um die Uebertragung des Sauerstoffs der Chromsäure auf die verbrennende Substanz augenscheinlich zu machen; in dem Verhältniss nämlich, als der chromsaure Baryt seinen Sauerstoff abgiebt, geht die Farbe desselben aus dem zeisiggrünen ins Dunkelgrüne über. Die Farbe des chromsauren Baryts wird wie die der meisten chromsauren Salze beim Erhitzen dunkler, sie wird selhst lebhaft dunkelorange, und nimmt beim Erkalten die urspūngliche Farbe wieder an. Um diese Verbrennungen auszuführen, wurde der chrom- 
304 Sace: Analysedes Samensdes weissen Mohns.

saure Baryt analysirt, da die Bestimmung desselben eine ausserordentliche Genauigkeit erforderte, so controlirte ich die Aequivalente des Chroms und Baryums und bediente mich zu diesem Zweck der chromsauren Salze des Baryts und des Qnecksilberoxydes, des chrom- und salpetersauren Baryts. Nachdem diese Verbindungen mehreremale und nach verschiedenen Methoden analysirt worden waren, erhielt ich als Mittel von vier Bestimmungen genau die Aequivalentzahlen von Berzelius.

Cortrole der Analyse der näheren Beslandtheile der Mohnsamen vermittelst der Asihenbestimmuny.

I. 3,1379 Grm. nicht getrocknete und normale Mohnsamen gaben 0,1697 Grm. einer grauweissen Asche.

II. 2,1838 Grrm. gaben 0,1173 Grm. Asche.

In 100 Theilen:

$$
\text { Asche } \underset{5,4080}{\stackrel{\text { I. }}{5,371 .}} \underset{5,3897}{\operatorname{Im}} \text { Mittel. }
$$

Bestimmung der Asche der trocknen Samen. Asche.

I. 1,4544 Grm. trockner Mohnsamen gaben 0,0988 Grm.

II. 2,6307 Grm. gaben 0,1896 Grm. Asche.

In 100 Theilen:

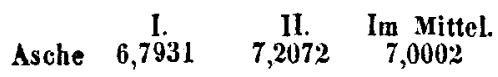

Nach diesen Resultaten enthalten die rohen Samen 29 p. C. Wasser, wäbrend durch direkten Versuch nicht ganz 25*p. C. gefunden wurden. Die Controle kann hier nicht in Betracht gezogen werden, da der geringste Fehler bei der Aschenbestimmung einen ungemein grossen bei dem Gewicht des Wassers nach sich zieht.

I. 4,1454 Grm. roher und feuchter Oelkuchen hinterliessen 0,3567 Grm. Asche.

II. 10,8260 Grm. hinterliessen 0,9435 Grm. Asche.

In 100 Theilen:

Asche $\quad \underset{8,6047}{\text { I. }} \quad \underset{8,7151}{\text { II. }} \quad \underset{8,6599}{\operatorname{lm} \text { Mittel. }}$

I. 2,7661, Grm. trockner Kuchen gaben 0,2967 Grm. Asche. 
Sacc: Analysedes Samens des weissen Mohns.

II. 3.8527 Girm. derselben Sulstanz gaben 0,4025 Grm. Asche.

In 100 Theilen:

$$
\text { Asche } \quad \underset{10,7263}{1} \quad \operatorname{ll}_{10,4472} \quad \operatorname{Im~Mittel.~}_{10,58675}
$$

Diese Zahlen erhöhen das Wasser in dem rohen Kuchen auf 22 p. C., sie erhöhen auch das Wasser auf 51 p. C. vom Gewicht des direkt aus den Mohnsamen ausgepressten Oeles, was durch den Versuch auf 45 p. C. festgesellt wurde.

1. 1,5305 Grm. mit Aether erschöpfter und getrockneter Substanz hinterliessen 0,1952 Grm. Asche.

I. 0,9581 Grm. hinterliessen 0,1308 Grm. Asche.

In 100 Theilen:

$$
\text { Asche } \quad \operatorname{II}_{12,7540} \quad \underset{13,6521}{\operatorname{Im}} \quad \underset{13,2031}{\text { Mittel. }}
$$

Diese Analysen setzen das Gewicht der in Aether löslichen Substanzen auf 24 p. C. lest, während durch den direkten Versuch nur 13 p. C. gefunden wurden.

I. 1,1222 $\mathrm{Grm}$. roher getrockneter Kuclsen mit der ungefähr 50 fachen Menge Wasser bei $1^{n}$ gerieben, vier und zwanzig Stunden lang digerirt und darauf ausgewaschen, hinterliessen 0,0975 Grm. Asche.

II. $1,5176 \mathrm{Grm}$. gewaschener liuchen hinterliessen 0,1356 Girm. Asche.

In 100 Theilen :

$$
\text { - Asche } \begin{gathered}
\mathbf{8 , 6 8 8 3} \\
\text { I. }
\end{gathered} \underset{\mathbf{8 , 9 3 5 1}}{\text { II. }} \underset{\mathbf{8 , 8 1 1 7}}{\text { Mittel. }}
$$

Die Zahlen sind um 1,7750 Grm. niedriger, als die bei der Aschenbestimmung des rohen, nicht gewaschenen Kuchens erhaltenen; sie künnen also nicht zur Controle der Menge des Albumins und Caseinns dienen, die der Kuchen an das Wasser abgiebt.

Das Albumin und Casein der Mohnsamen enthält demnach mineralische Substanzen, die sich mit den Proteînsubstanzen im Wasser lósen

I. 0,1978 Grm. Holzfaser von der Behandlung der Holzfaser mit Schwefelsảure und Soda herrührend, hinterliessen $0,0080 \mathrm{Grm}$. Asche.

Joura. f. prakt. Cheraie. XLIX. 5. 
II. 0,125j Gim. derselben Substanz hinterliessen 0,0048 liril. Asche.

In 100 Theilen:

$$
\text { Asche } \underset{4,0545}{\text { I. II. }} \quad \underset{3,8255}{\operatorname{Im} \text { Mittel. }}
$$

Die Asthe ist vollkommen weiss und besteht aus Kieselerde und schwefelsanrem Kalk. Letzterer ist jedenfalls durch die Belaandlung mit Schwelelsäure entstanden.

Die vorstehenden Analysen zeigen, dass die Aschenbestimmuny uicht zur Controle der Analysen angewendet werden kann, weun ihr Gewicht bei Luftzutritt bestimmt wird; es ist möglich, dass man mit der ror kurzer Zeit von Mitscherlich entdeckten, sehr vollkommenen, aber auch ausserordentlich schwierigen Vlethode bessere Resultate erreichen wird.

Ueber die Art der Vertheilung der mineralischen Bestandtheile in dem Innern der Samenkorner ist nichts Neues aufyefunden worden.

\section{Aschenanalyse.}

Vie angewendete Asche war durch Verbrennen des Kuchens dargestellt worden; sie war granweiss und enthielt etwas Kohle. Sie enthielt weder Eisen, noch Mangan, Thonerde und Chlor.

I. 2,3789 Grn. Asche gaben 0,1238 Grm. Kieselerde 1,1899 kohleusauren Kalk, 0,2933 Grm. pyrophosphorsaure Talkerde, 1,5595 Grm. phosphorsaures Eisenoxyd.

II. 2,7654 Grm. Asche gaben 0,1342 Grm. Kieselerde, 1,3903 Grm. kohlensauren Kalk, 0,3138 Grm. pyrophosphorsaure Magnesia und 1,8421 Grm. phosphorsaures Eisenoxyd.

III. 1,6497 Grm. Asche gaben 0,0737 Grm. Kieselerde und 0,0957 Grm. schwefelsauren Baryt.

IV. 2,8839 Grm. Asche gaben 0,3801 Grm. Chloralkalien und 0,0875 Kaliumplatinchlorid.

In 100 Theilen: 
Sacc: Analyse des Samens des wussen Mohns. 307

Kieselerde

Schwefelsänre

Phosphorsäure

Talkerde

Kalk

Natron

Kali

Kohlensäure durch Differenz

$\begin{array}{ccccc}\text { I. } & \text { II. } & \text { III. } & \text { IV. } & \text { Im Mittel. } \\ \mathbf{5 , 2 0} & 4,85 & \mathbf{4 , 4 6} & - & 4,84 \\ \overline{37,51} & \mathbf{3 8 , 1 1} & 1,99 & - & 1,99 \\ 4,51 & 4,15 & - & - & 37,81 \\ 28,00 & 28,15 & - & - & 4,33 \\ - & - & - & \mathbf{4 , 4 7} & 28,08 \\ - & - & - & \mathbf{0 , 8 2} & 0,87 \\ - & - & - & - & 17,66 \\ & & & & 100,00 .\end{array}$

Controle der Resullate der Analyse der näheren Bestandtheile durch die Verbrennung.

Wenn man für das Mohnül die oben angegebene Zusammensetzung, für das Proteïn die Zusammensetzung

\begin{tabular}{|c|c|}
\hline Kollensteff & \\
\hline Wasserstoff & \\
\hline Stickstoff & $\begin{array}{l}0,04 \\
16,34\end{array}$ \\
\hline Samerstoff & 20,94 \\
\hline & \\
\hline
\end{tabular}

uni für die Holzfaser, das Bassorin oder die Pektinsänre die Zusammensetzung

$$
\begin{array}{lr}
\text { Kohlenstoff } & 44,78 \\
\text { Wasserstoff } & 5,21 \\
\text { Sauerstoff } & 50,01 \\
\cline { 2 - 2 } & 100,00 .
\end{array}
$$

anniunmt, so lässt sich berechnen, dass die Molnnsamen folgendermassen zusammengesetzt sind:

\begin{tabular}{lrr} 
& Berechnet. & \multicolumn{1}{c}{ Gefunden. } \\
Kohlenstoff & 61,3382 & 62,2338 \\
Wasserstoff & $\mathbf{8 , 7 5 0 7}$ & $\mathbf{9 , 2 0 1 4}$ \\
Stickstoff & $\mathbf{1 , 7 2 0 5}$ & $\mathbf{3 , 5 9 2 4}$ \\
Sauerstoff & $\mathbf{2 1 , 1 9 0 4}$ & $\mathbf{1 7 , 9 7 2 2}$ \\
Asche & 7,0002 & $\mathbf{7 , 0 0 0 2}$ \\
\cline { 2 - 3 } & 100,0000 & $100,0000$.
\end{tabular}

Die berechneten Zahlen stimmen ziemlich mit den gefundenen überein, davon ausgenommen ist der Stickstoff, dessen Menge berechnet genau die Hälfte von der gefundenen Quantitảt beträgt. Diese Differenz kann nur davon herrühren, dass sich der Stickstoff in den Körnern als Amid oder Ammoniak mit Pektinsäure, Proteĩn und Phosphorsāure verbunden hefindet. An der Gegenwart des Ammoniaks, in den Hohnsamen ist kaum zu zweifeln, da sich eine beträchtliche Menge dessellen entwickelt, wenn man die Samen mit Aetznatron zerreibt. 
Der berechnete Kohlenstoff ist etwas geringer als der gefundene; dies kommt daber, dass die Zusammensetzung der in den Samen enthaltenen flüchtigen Substanzen nicht bekannt war; es wurde denselben aber die des fetten Oeles zugeschrieben, das reich an Wasserstoff ist und wenig Sauerstoff enthält $\left(\mathrm{C}_{9} \mathrm{H}_{y} \mathrm{O}\right)$, wãhırend die flüchtigen Substanzen müglicherweise aus kohlenstofreicheren Kohlenwasserstoffen bestehen konnten.

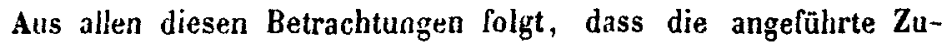
sammensetzung der Mohnsamen richtig ist.

Der rohe und trockne Kuchen besteht aus :

\begin{tabular}{|c|c|c|}
\hline $\begin{array}{l}\text { Kohlenstoff } \\
\text { Wasserstoff } \\
\text { Stickstoff } \\
\text { Sauerstoff } \\
\text { Asche }\end{array}$ & $\begin{array}{c}\text { Berechnet. } \\
41,78 \\
4,95 \\
4,29 \\
33,78 \\
13,20 \\
\end{array}$ & $\begin{array}{c}\text { Gefunden. } \\
42,27 \\
6,04 \\
7,64 \\
30,85 \\
13,20 \\
\end{array}$ \\
\hline & $\overline{100,00}$ & 100,00 \\
\hline
\end{tabular}

Viese Zablen zeigen, dass entweder freies Ammoniak oder eins seiner Derivate in den Mohnkornern enthalten ist, denn in dem Masse, ais der Kuchen fettes und atherisches Oel verioren hat, nimmt der Verlust des Stickstoffs und Wasserstoffs zu, welche beiden Körper in anderer Form als in Gestalt von Proteĩn vereinigt sein müssen.

Es ist gewiss nicht uninteressant zu wissen, dass der Stickstoff in den Kōrnern in wägbarer Menge in anderer Form, als in den organischen Verbindungen rorkommen kann, weil, wenn dieses Faktum auf alle stickstoffhaltigen organischen Substanzen anwendbar ist, man aus ihrem Stickstoffgehalt keinen Schluss auf ihren Werth als Nahrungsmittel ziehen kann, da ein Theil des Stickstoffs in Gestalt einer mehr oder weniger wasserstoffreichen Verbindung, die nicht assimilirbar ist, und durch Basen ausgetrieben werden kann, darin enthaiten ist.

Es bleibt nun noch zu untersuchen, ob in den Mohnsamen ebenso wie in allen āhnlichen Samenkőrnern Pektin und Protein oder was gleich ist Pektinsãure und Proteinsảure in Form von Ammoniaksalzen, oder von Amid - und Imidverbindungen vorkommen, die sich beim Zusammenbringen mit Alkalien. in lósliche alkalische Salze und sich entwickelndes Ammoniak zersetzen. 